\title{
Androgen deprivation therapy (castration therapy) and pedophilia: What's new
}

\author{
Mauro Silvani, Nicola Mondaini, Alessandro Zucchi \\ ${ }^{1}$ Urologist in chief, Urology Dept. "Degli Infermi” Hospital, Biella, Italy; \\ ${ }^{2}$ Urologist, Urology Unit, Santa Maria Annunziata Hospital, Firenze, Italy; \\ ${ }^{3}$ Urologist, Urology and Andrology Dept. University of Perugia, Perugia, Italy.
}

\begin{abstract}
Summary Andrology is a constantly evolving discipline, embracing social problems like pedophilia and its pharmacological treatment. With regard to chemical castration, the andrologist may perform an important role as part of a team of specialists. At present, no knowledge is available regarding hormonal, chromosomal or genetic alterations involved in pedophilia. International legislation primarily aims to defend childhood, but does not provide for compulsory treatment. We reviewed international literature that, at present, only comprises a few reports on research concerning androgen deprivation. Most of these refer to the use of leuprolide acetate, rather than medroxyprogesterone and cyproterone acetate, which present a larger number of side effects. Current opinions on chemical castration for pedophilia are discordant. Some surveys confirm that therapy reduces sexual thoughts and fantasies, especially in recidivism. On the other hand, some authors report that chemical castration does not modify the pedophile's personality. In our opinion, once existing legislation has changed, andrologists could play a significant role in the selection of patients to receive androgen deprivation therapy, due in part to their knowledge about its action and side effects.
\end{abstract}

KEY WORDS: Pedophilia; Chemical castration; Andrology.

Submitted 30 April 2015; Accepted 30 July 2015

\section{INTRODUCTION}

Andrology is a constantly evolving discipline, embracing social and legal problems such as pedophilia. There is no clear-cut definition of pedophilia: it is not easy to place it within a conceptual framework due to the difficulty in bringing the two orientations - socio-anthropological and clinical - together in a "single concept".

In socio-anthropological terms, pedophilia is a form of social perversion that is only classed as such in certain civilizations and during certain periods of history (1); child sexual abuse has always existed in all populations. It is not a phenomenon associated with so-called "complex societies", nor can it be linked to modernity. However, to date, there are no known specific chromosomal, genetic, hormonal alterations or neurological diseases associated with pedophilia, though some authors suggest a genetic predisposition $(2,3)$.

The criteria used for the clinical diagnosis of pedophilia are usually taken from the DMS-V (4). International legislation primarily aims to defend childhood, but does not provide for compulsory treatment. Pharmacological solutions with andrological implications, such as "chemical castration" (Androgen Deprivation Therapy - ADT), have been proposed for the clinical management of pedophiles. Inevitably, this therapeutic option poses major legal and moral implications (5), but current literature does not provide any indications. For this reason, we reviewed international literature that, at present, only comprises a few reports on research concerning ADT in pedophilic patients, considering the psychological aspect of the disease and leaving out the crucial role of the andrologist. Furthermore, we performed a revision of current legislation in more developed Western countries.

\section{Materials AND METHOdS}

We performed a detailed review of literature about chemical castration using ADT, also examining the effects of each of these drugs and the side effects of androgen deprivation.

Rationale for chemical castration in pedophiles In all probability the term "castration" derives from the Latin word castor or beaver. The therapeutic benefits of beaver testicles were described by Galen and Pliny, who urged people to hunt this animal. Today, this term implies a therapeutic approach designed to inhibit sexual impulses or block them with specific chemical compounds. Chemical castration is therefore the common term, but it would be more appropriate to speak of "testosterone antagonist pharmacological therapy". The effects of pharmacological castration are a reduction in libido, sexual fantasies, frequency of masturbation and pleasure, erections (both nocturnal and induced by erotic stimuli), blood testosterone, FSH and LH, and an increase in prolactin (6).

There are a number of different reasons for androgen deprivation therapy (ADT) in pedophile patients:

- reduction and elimination of the most significant symptoms of the sexual malaise;

- controlling the ideational, repetitive and aggressive threat to minors;

- reducing sexual attitudes and urges to the point of eliminating them; 
- easier application of psychotherapy and cognitive behavioral therapy;

- low percentage (just 3-5\%) of pedophile recovery with psychotherapy alone;

- possible association with other paraphilias (such as sadomasochism) (6-10).

However, certain currents of thought are completely opposed to this procedure. MOIGE (an Italian parents' movement - www.moige.it) has a negative view of chemical castration, claiming that it leads to more aggression in the subject when therapy is suspended and that it does not change the pedophile's personality. Moreover, the pedophile may accept it simply to avoid detention. Lastly, in particular cases, it is contraindicated due to potential side effects (anemia, osteoporosis, etc.). It is also important to consider other fundamental aspects. Long-term treatment is intended to reduce the symptoms and is not a cure. Moreover, no controlled studies have been conducted on the long-term efficacy of androgen deprivation therapy. The pedophile commits the crime for reasons that go beyond pure sexual gratification; as a result, reducing libido does not necessarily mean eliminating the risk of repeat offences (10). Furthermore the pedophilic act does not necessarily require an erection or penetration.

The following drugs are used in chemical castration:

1. cyproterone acetate;

2. diethylstilboestrol (no longer in use);

3. medroxyprogesterone acetate;

4. LHRH analogues: particularly leuprolide acetate (11-13)

Medroxyprogesterone acetate (MPA) was first used in pedophilia therapy in 1990 by Cooper at al. (14). The drug was administered at a dose of $300 \mathrm{mg}$ per day for a period of two years. After two weeks of treatment, the authors observed a $73 \%$ reduction in total blood testosterone, a $300 \%$ increase in blood prolactin, and a noticeable reduction in nocturnal and stimulation-induced erections that persisted even two months after stopping treatment and the recovery of physiological blood testosterone levels. However, this study is limited due the fact that it involved just six patients. Maletzky carried out a broader study in 2003, with essentially comparable results $(8,15)$. The risks associated with the prolonged use of this drug, as emphasized by Kruger et al. in a recent study conducted in 2006, are metabolic in nature. In fact, over time a reduction in carbohydrate tolerance is observed due to an increase in basal insulin levels. The drug also stimulates lipoprotein lipase, producing a tendency to obesity and, by reducing HDL levels, it significantly increases atherogenic risk. Moreover, a reduction in the effects of aldosterone on the renal tubules is described, with diminished sodium reabsorption and increased secretion of glucocorticoids by the adrenal cortex. Cases of Cushing's syndrome and adrenocortical insufficiency have been described. The drug also increases the mitotic activity of the acinar epithelium of the breast, leading to a risk of gynecomastia and breast cancer (16). Another drug used was cyproterone acetate (CPA). Despite the fact that they date back to 1992 (17), the studies conducted by Cooper et al. provide interesting data. The study used a dose of $100 \mathrm{mg}$ /day over an average period of 28 months. After two weeks of taking the drug, a reduction in testosterone, $\mathrm{FSH}$ and $\mathrm{LH}$, and an increase in prolactin were observed. Sexual urges were also reduced in parallel with testosterone levels. The effect on diurnal erections varied from subject to subject. When therapy was suspended, testosterone levels rose rapidly. The authors also conducted a controlled double-blind study to assess the effects of CPA and MPA, and found that they were comparable and dose-dependent. Another study by Lasket (18) shows that the broadest studies of the drug occurred in Germany, where benefits in 100 male pedophiles, sadists and exhibitionists included decreased sexual drive, lower frequency of orgasms and less frequent erections. The drug most commonly used today is an LHRH agonist: leuprolide acetate $(11,12)$.

Published studies demonstrate that analogues are more effective on the sexual sphere than MPA and CPA, and on sexual urges, desire, ejaculation, and erections and orgasms, but with fewer side effects $(13,14,16,17,19)$. The literature only includes a very small number of case studies regarding the use of leuprolide in the treatment of pedophiles. One of the most significant studies is the one conducted by Briken et al., which recruited eleven men aged between 41 and 63, all of whom repeat offenders. All the subjects were treated for one year, with a reduction in aggressive sexual behavior, erections and masturbation. One of the subjects committed suicide (20). Another interesting study is the one conducted by Schober et al. (21), assessing five pedophiles treated for a year with quarterly leuprolide acetate. The reduction in testosterone, FSH and LH was rapid, as was the increase in blood prolactin. There was a reduction in urges, erotic fantasies and nocturnal erections assessed with the Rigiscan test. After one year of therapy, the penile circumference at the base of the shaft had decreased from an average of 9.7 to $8 \mathrm{~cm}$. The number of case studies is noticeably larger if we also consider patients with different paraphilias. In fact, the work carried out by Briken et al. in 2003 (20) assesses 118 patients treated for 6-24 months and affected by different types of paraphilias. In some cases, SSRIs (selective serotonin reuptake inhibitors) were also used without any evidence of better success. Literature also includes an interesting study conducted by Almeida et al. in 2004, which demonstrates that the drastic reduction in testosterone levels leads to a highly neurotoxic increase in beta-amyloid protein associated with the pathophysiology of Alzheimer's disease. (22). Finally no randomised controlled trials have yet been published (23).

As to non-androgen deprivation pharmacological therapy designed to treat the psychiatric disorder, various categories of psychoactive drugs can be used $(24,25)$ :

- tricyclic antidepressants (clomipramine and desipramine);

- SSRIs, paroxetine, sertraline, etc.;

- typical and atypical neuroleptics, benperidol, haloperidol, thioridazine;

- benzodiazepines (diazepam, clonazepam, etc.);

- lithium salts, lithium carbonate;

- antiepileptics, carbamazepine.

Antidepressants can be used to reduce libido and delay ejaculation to the point of anorgasmy, especially in the event of comorbidity with mood disorders and obses- 
sive-compulsive disorder. SSRIs offer advantages in the treatment of paraphilia because they permit simple monitoring of the patient, even on an outpatient basis, and form a valid option together in combination with psychotherapy.

\section{Possible pharmacological therapies pertaining to the andrologist}

In definitive terms, pedophilia therapy is comparable to medical therapy for prostate cancer and andrologists usually work with therapy for the male sex, including androgen deprivation. It should be remembered that the therapy can obviously be combined: androgen deprivation and SSRIs, benzodiazepine (clonazepam) or carbamazepine. These combinations must obviously come after psychological and/or psychiatric assessment.

\section{Current legislation in Italy}

The first official Italian proposal regarding chemical castration was made in Milan on 17 February 1997 during the trial of a man accused of repeat rape. Since then, there has been much disagreement about the process. However, it should be remembered that in Italy no one can be forced to receive medical treatment unless so ruled by law. Moreover, the law cannot violate the limits imposed by the need to respect the individual, as enshrined in Article 32 of the Italian Constitution, paragraph II. The National Bioethics Committee specifies that "the infrangibility of the human body is always valid even in convicted criminals, unless expressly requested by the individual concerned".

In Italy, crimes linked to pedophilia are governed by Law $269 / 98$, as updated in 2006, which regulates against pedophilia, the exploitation of prostitution, pornography, sex tourism involving minors and new offences of slavery. This law exclusively regards means for repressing crime linked to pedophilia, but excludes the possibility of intervening with suitable pharmacological therapy. In greater detail, the primary objective of the law is to protect children against all forms of sexual exploitation and violence, safeguarding their physical, psychological, moral and social development. In addition to the cited law, crimes of pedophilia are regulated by Law 66/96 containing the new regulations against sexual violence. This law finally repealed the provisions of the Rocco Code, meaning that the crime of sexual violence is no longer considered a crime against public morality and decency, but is instead a crime against a person.

Law 66/96 finally interprets the sexual act objectively for the first time: assessed in relation to the objectifying nature of the sexual act, determined according to medical-psychological and anthropological-sociological indications. Therefore, sexual relevance is configured in relation to the local customs.

\section{Chemical castration and legislation in other European countries}

In Germany, chemical castration has been legal since 1969. It is only permitted in individuals aged over 25 , once a final sentence has been passed and after they have undergone clinical assessment regarding their suitability for treatment. In Denmark the provision has been in place since 1973 and has replaced surgical castration, approved since 1929. Those who undergo this treatment benefit from early release or a shorter sentence. Social services monitor the implementation of the pharmacological castration program.

In Sweden it has been in place since 1993 and is only applicable if requested by the person concerned, in the case of a high risk of recidivism. Those who undergo the treatment receive certain benefits and shorter sentences. In France it has been permitted under state law since 1997, but only for those who volunteer to receive it; it was first applied in 2005. Nicolas Sarkozy adopted a very restrictive position regarding the phenomenon of pedophilia over recent years, according to which a significant role is filled by androgen deprivation therapy together with more severe prison sentences $(26,27)$. The first European pedophile center was slated to open in Lyon in 2009. Pedophiles are assessed by a panel of specialists, responsible for examining the risk level posed by each individual on a case by case basis.

In the United Kingdom chemical castration can be administered on the direct request of those convicted of crimes of pedophilia. Since 7 January 2008, all those condemned for sexual abuse offences have been informed of the possibility of receiving chemical castration at their own request. However, there is no monitoring to ensure that these patients comply with the oral or injection therapy, which has led to many objections being raised by various associations, both for and against chemical castration. In Norway, pharmacological treatment of those convicted of sex crimes has been possible, subject to consent, since 2004, together with group psychological therapy. In this case, castration does not lead to reduced sentences or other privileges.

In Spain, research is being carried out into psychological and pharmacological measures other than chemical castration that could reduce libido in pedophile patients.

\section{Chemical castration: legislation in the USA}

On 17 September 1996, California became the first American state to issue a law providing for chemical castration of pedophiles. The drug authorized for this purpose is medroxyprogesterone acetate (MPA). The state pays only half of the cost of therapy, while the offender is responsible for the other half. The treatment is not subject to any age limits. The patient is informed of the side effects of the treatment, but the therapy can only be authorized at the discretion of the ruling court. In the state of Florida, the law on chemical castration came into effect on 1 October 1997. The treatment is administered after the second pedophilia offence and the court decides the duration of the treatment. The offender is not informed of the therapy he is receiving, nor of the side effects. Instead, the suitability of treatment is decided by a medical team.

\section{Chemical castration: legislation in Korea}

In 2011 the Ministry of Justice of Korea enacted legislation requiring chemical castration of paraphilic sexual offenders over the age of 19 and/or those at high risk of recidivism. The current chemical castration legislation calls for the psychiatric evaluation of sex offenders and 
chemical castration is performed 3 months prior to probation so that the treatment may effectively change the behavior of the offender (28).

\section{Discussion}

A scientific publication such as this one on pedophilia may seem unusual but, in fact, statistical data, such as the findings of 1994 (Caffarra et al.) and CENSIS data (1999), reveal that 470 cases of child sexual abuse are reported every year in Italy, including 64 by unknown offenders (www.pacse.censis.it) $(29,30)$. The most commonly involved age bracket is between 11 and 14 years (9). The estimated annual incidence of sexual abuse is: - 1 case per 400 minors; - 1 case per 4 schools; - 1 case per 500 families.

Official Italian news reports indicate a constant and considerable rise in the number of reported cases of child abuse on a yearly basis (26).

In the first half of 2003, compared to the same period in 2002 , this type of violence saw a $62.7 \%$ increase in victims in the 11-14 age bracket and a $43.9 \%$ increase in the age bracket of 10 and under (www.etica2001.com/ Monitoraggio Castelfranco.asp), with the overall number of victims rising from 296 to 438. It should also be borne in mind that the ratio of reported to non-reported offences is 1 to 100 . The research carried out by Laederach at 68 schools in Geneva on a sample of 1,193 adolescents concluded that $20.4 \%$ of girls and $3.3 \%$ of boys had experienced abuse of a sexual nature before the age of 14 (31). The problem of pedophilia is certainly being placed increasingly in the spotlight. The media constantly brings reported cases to public attention and it is therefore a subject of discussion on all levels, legal, social, moral, medical, and scientific alike. What role can the andrologist play in the treatment of pedophiles? It should be remembered that over the last twenty years the treatment of pedophiles has been entrusted to psychiatrists, psychologists, and sexologists: this is certainly a multidisciplinary approach.

However, the andrologist has detailed knowledge of the mechanisms of action and the side effects of the drugs used in androgen deprivation, which are the same drugs used to treat prostate cancer. Obviously, this does not exclude the assessment of suitability for pharmacological castration formulated by other medical figures, endocrinologists, psychiatrists, criminologists, and so on. The threshold below which androgen deprivation therapy should not be administered could be set at 25 because, at this age, the hypothalamic-pituitary-gonadal axis has reached maturity. Castration should be continued for a sufficiently long period of time - at least two or three years - in combination with psychotherapy or SSRI therapy, until the subject's obsessive-compulsive disorder and/or repetitive sexual ideations are under control.

As clearly shown in a recent paper by Amelung et al. (32), there are few randomized study about chemical castration with ADT and this retrospective study presents initial data on self-motivated, help-seeking pedohebephiles choosing ADT in the course of a psychotherapy program. Furthermore, this study clearly showed a decrease in paraphilic sexual behavior and offense-supportive cognition under combined psychotherapy and ADT, although only 15 patients agreed this therapy out of a total of 111 patients. Unfortunately, there were different factors leading to discontinuation/non-acceptance of therapy: in this study, both the intended and the adverse effects of the medication seemed to influence compliance.

In daily practice, only a small number of pedophiles voluntarily request treatment and they reach this decision because they experience anxiety or depression associated with this perversion; otherwise they do not spontaneously renounce their sexual orientations.

For these reasons, careful education and monitoring of patients interested in ADT regarding the potential effects and side effects is fundamental. In any case, as noted, the medical therapy used for androgen deprivation in pedophiles is the same therapy used to treat prostate cancer: it is important to consider the effects of therapy with LHRH super-agonists on a systemic level, such as osteoporosis, increased physical fatigue, gynecomastia, depression. and anemia. Though some of these adverse effects occur regardless of the means of castration, GnRH agonists may achieve lower testosterone levels while having fewer side-effects than CPA and MPA; however, the risk of bone demineralization remains and may require additional treatment (23). Nevertheless, according to the World Federation of Societies of Biological Psychiatry (WFSBP), when properly administered, with an appropriate protocol in place to detect and treat side effects should they develop, GnRH agonist treatments constitute no more or less of a risk than other pharmacological agents and represent the most promising treatment in pedophilic patients (23). From this point of view the andrologist is a highly specialized professional figure in a position to assess any indication/contraindications present in pedophile candidates for androgen deprivation therapy, as part of a team of specialists. However, it is important to examine certain considerations regarding the treatment of pedophiles: many believe that the behavior they have adopted is innocuous and they only agree to treatment to avoid detention. Moreover, during their lives they have found an erotic solution to their problems and, as a result, they are rarely interested in giving it up. Many psychiatrists believe that the most valid approach is insight-oriented psychotherapy; through these processes the patients become aware of the dynamics and events that caused the pedophilia to develop, improving their self-esteem and interpersonal skills, and helping them find more acceptable methods of sexual gratification. In any case, the current legislation of Western countries is deficient and is primarily focused on protecting younger victims of sexual abuse. As a result, it does not support the clinical activity of the clinicians (andrologists, psychiatrists, etc.) but merely places ethical restrictions.

\section{Conclusions}

Pedophilia is an illness as well as a legal and social phenomenon with criminal implications. Detention and psychotherapy alone are not sufficient to control the phenomenon and prevent repeat offences, as detention is not long enough to counteract the phenomenon.

In patients who have been sentenced and have a psycho- 
logical profile strongly suggestive of potential recidivism, chemical castration could provide a significant therapeutic option. Aside from the severity of the phenomenon in the face of public opinion, a political class and a scientific world largely divided between those for and those against chemical castration, there are questions that every doctor who deals with the problem must ask. Would it be desirable to have a law that provides for compulsory pharmacological treatment without the consent of the person concerned? Lastly, prevention plays a significant role in the phenomenon of pedophilia, necessarily involving the institution, the family and, above all, schools; children spend many hours at school and teachers need to be able to interpret any signs of discomfort that may be manifested. Science and institutions remain strongly divided regarding the issue of androgen deprivation in pedophiles, partly because its adoption would certainly open up a significant precedent for application in all other forms of sexual violence, especially against women.

\section{REFERENCES}

1. De Masi F. The paedophile and his inner world: theoretical and clinical considerations on the analysis of a patient. Int J Psychoanal. 2007; 88:147-65.

2. Alanko K, Salo B, Mokros A, Santtila P. Evidence for heritability of adult men's sexual interest in youth under age 16 from a populationbased extended twin design. J Sex Med. 2013, 10:1090-9.

3. Mendez M1, Shapira JS. Pedophilic behavior from brain disease. J Sex Med. 2011; 8:1092-100.

4. American Psychiatric Association. DSM V - Diagnostic and Statistical Manual Disorders. Washington, DC: American Psychiatric Publishing, Inc. 2013.

5. Berti A, Maberino C. Pedophilia: do effective treatments exist? Giorn It Psicopat. 2002; 8:14-8.

6. Houts FW, Taller I, Tucker DE, Berlin FS. Androgen deprivation treatment of sexual behavior. Adv Psychosom Med. 20121; 31:149-63.

7. Drapeau M, Korner A, Granger L, et al. A plan analysis of pedophile sexual abusers' motivation for treatment. A Qualitative pilot study. Int J Offender Ther Comp Criminol. 2995; 49:308-24.

8. Maletzky BM. The biological treatment of dangerous sex offenders. A review and preliminary report of the Oregon pilot depo-Provera program. Arch Sex Behav. 2003; 8:391-412.

9. Heim N. Sexual behavior of castrated sex offenders. Arch Sex Behav. 2005; 10:9-11.

10. Hughes JR. Review of medical reports on paedophilia. Clin Pediatr Phil. 2007; 14:342-55.

11. Iustine M, Schober L, Peter M. Leuprolide acetate is a eamiliar drug that may modify sex-offender behaviour: The Urologist's role. BJU International. 2006; 97:684-6.

12. Schober JM, Kuhn PJ, Kovacs PG, et al. Leuprolide acetate suppresses pedophilic urges and arousability. Arch Sex Behav. 2005; 34:691-705.

13. Briken P, Nika E, Berner W. Treatment of paraphilia with luteinizing hormone-releasing hormone agonist. J Sex Marital Ther. 2001; 27:45-55.

14. Cooper AJ, Sandhu S, Losztyn S. A double-blind placebo controlled trial of MPA and CPA with seven pedophiles. Can J. Psychiatry. 1992; 37:687-93.

15. Hill A, Briken P, Kraus C, et al. Differential pharmacological treat- ment of paraphilias and sex offenders. Int J Offender Ther Comp Criminol. 2003; 47:407-421.

16. Krueger RB, Hembree W, Hill M. Prescription of medroxyprogesterone acetate to a patient with pedophilia, resulting in Cushing's syndrome and adrenal insufficiency. Sex Abuse. 2006; 18:227-8.

17. Cooper AJ, Cernovovsky Z. The effects of CPA on sleeping and waking penile erections in pedophiles: possible implications for treatment. Can J Psychiatry. 1992; 37:33-9.

18. Laschet V, Laschet L. Psychopharmacotherapy in sex offenders with CPA. Pharmakopsychiatr Neuropsychopharmakol 2001;4:99-104.

19. Vanderschueren DM. Hormone treatment of pedophilia. Geneeskd Belg. 1997; 59:371-8.

20. Briken P, Hill A, Berner W. Pharmacotherapy of paraphilias with long-term agonist of luteinizing hormone-releasing hormone: A systematic review. J Clin Psychiatry. 2003; 64:890-7.

21. Schober JM, Kuhn PJ, Kovacs PG, et al. Leuprolide acetate suppresses pedophilic urges and arousability. Arch Sex Behav. 2005; 34:691-705.

22. Almeida OP, Waterreus A, Spry N, et al. One year follow-up study of the association between chemical castration, sex hormones, betaamyloid, memory and depression in men. Psychoneuroendocrinology. 2004; 29:1071-81.

23. Thibaut F, De La Barra F, Gordon H, et al. The World Federation of Societies of Biological Psychiatry (WFSBP) Guidelines for the biological treatment of paraphilias. The World Journal Biol Psychiatry. 2010; 11:604-655.

24. Prahlada Rao N, Chand PK, Murthy P. A case of late-onset pedophilia and response to sertraline. Prim Care Companion J Clin Psychiatry. 2007; 9:235-6.

25. Cantor JM, Kabani N, Christensen BK, et al. Cerebral white matter deficiencies in pedophilic men. J Psychiatric Res. 2008; 42:167-83.

26. Pellai A, Castelli B, Scyvlowska G, Bassoli L, et al. Child sexual abuse primary prevention: outcome evaluation of a health education project implemented in Milan's elementary schools. Ann Ig. 2003; 15:529-39.

27. Nau JY. Nicolas Sarkozy and pedophilia. Rev Med Suisse 2007; 3:1980.

28. Koo KC, Ahn JH, Hong SJ, et al. Effects of chemical castration on sex offenders in relation to the kinetics of serum testosterone recovery: implications for dosing schedule. J Sex Med. 2014; 11:1316-24.

29. Caffarra D. Uscire dal silenzio. Gli abusi sessuali e l'incesto. ASPER; CENSIS, 1998.

30. Centro Studi Investimenti Sociali (CENSIS). Sfruttamento sessuale e minori: nuove linee di tutela. Un progetto contro l'abuso sessuale, c.r. 11641. Rome 1998

31. Bouvier P, Halpérin D, Rey H, et al. Typology and correlates of sexual abuse in children and youth: multivariate analyses in a prevalence study in Geneva. Child Abuse Negl. 1999; 23:779-90.

32. Amelung T, Kuhle LF, Konrad A, et al. Androgen deprivation therapy of self-identifying, help-seeking pedophiles in the Dunkelfeld. Int $J$ Law Psychiatry 2012; 35:176-84.

\section{Correspondence}

Mauro Silvani, MD Urologist in chief

Urology Dept. "Degli Infermi” Hospital, Biella, Italy

Nicola Mondaini, MD Urologist

mondatre@hotmail.com

Urology Unit, Santa Maria Annunziata Hospital, Firenze, Italy

Alessandro Zucchi, MD Urologist

Urology and Andrology Dept. University of Perugia, Perugia, Italy 